\title{
Sexualité, échange de pouvoir et science-fiction : Une étude SMiotique de quelques textes de science-fiction québécoise ${ }^{1}$
}

\author{
SYLVIE BÉRARD \\ Trent University
}

\section{Résumé}

Cet article s'attarde à trois textes de science-fiction québécoise contemporaine mettant en scène une interaction sadomasochiste: « La carte du tendre » d'Élisabeth Vonarburg, Lame d'Esther Rochon et La mue de I'hermaphrodite de Karoline Georges. Combinant entre autres l'analyse du discours narratif, les théories psychanalytiques, et l'étude de la performance, il s'intéresse non seulement à ce que la science-fiction nous dit de la sexualité et du sadomasochisme, mais il tente de cerner ce que le sadomasochisme, en tant que cadre d'analyse, peut nous apprendre à propos de l'écriture de science-fiction et, pourquoi pas, à propos de l'écriture en général.

\begin{abstract}
La vérité vraie est que j'ai passé la plupart de mon adolescence - et, je dois l'admettre, de mes vingt ans - à me masturber en lisant des livres de science-fiction, de merveilleuses, impossibles histoires pleines de lutte et d'angoisse. (Allison 101)
\end{abstract}

Un jeune garçon doit décider s'il accepte, par amour, de porter dans son corps les larves d'une créature insectoïde dont l'espèce, dans son monde, vit en symbiose avec les êtres humains ${ }^{2}$; une jeune fille handicapée, reliée neurologiquement à un vaisseau spatial auquel elle sert de cerveau central, est confrontée à l'impossibilité de vivre une expérience charnelle avec son pilote ${ }^{3}$; des astronautes stérilisés pour des raisons de sécurité font l'objet d'une fétichisation au sein d'une communauté terrestre ${ }^{4}$; le corps humain est qualifié de «viande» («meat») par des adeptes du cyberespace qui lui préfèrent leurs identités virtuelles ${ }^{5}$ : voilà seulement un échantillon du traitement que la science-fiction est capable de faire subir au corps. Depuis l'impossible union entre l'humain et I'Autre pour des raisons d'incompatibilité physique jusqu'à l'emprisonnement de l'esprit dans la machine, de la mutation physique à la disparition pure et simple de l'enveloppe physique dans les univers virtuels, la sciencefiction n'est pas tendre avec le corps. Au gré des récits, elle lui fait subir toutes sortes de transformations, de mutations, de vexation ${ }^{6}$.

La sexualité en science-fiction ne va pas sans une certaine torture des corps, et, en matière de sexualité, la cruauté semble bien s'accommoder du contexte science-fictionnel. Aussi n'est-il pas trop étonnant, un cran plus loin, de retrouver tout un sous-genre qui combine la science-fiction (SF) et le sadomasochisme (SM). Ainsi, la série Gor de l'États-unien John Norman, située dans un monde science-fictionnel et mettant en scène des rapports de domination et d'esclavage, a créé une telle impression que des fans, se baptisant « goréens », se sont mis à vivre selon les règles établies dans la série (cf. Macintyre 2006); dans le monde virtuel Second Life, des régions entières sont d'ailleurs consacrées au « goréanisme » érigé en mode de vie. Le lecteur et/ou la lectrice peuvent bien sûr 
s'approprier le genre littéraire à toutes les fins, y compris à celle de l'excitation érotique, comme le montre l'épigraphe de cet article. Cependant, les deux champs (SF et SM) semblent aussi attirer en partie un public semblable, comme le souligne Cecilia Tan, anthologiste et auteure de fiction érotique et de science fiction ${ }^{7}$, qui dit trouver les mêmes livres (Histoire d'O ou classiques de la science-fiction) lorsqu'elle se rend dans les fêtes privées des membres des deux communautés ${ }^{8}$.

D’après Susan Sontag, il y aurait certaines similitudes entre le récit pornographique et celui de sciencefiction. Selon elle, le corps sadien, se conçoit « as a machine and [...] the orgy as an inventory of the hopefully infinite possibilities of several machines in collaboration with each other ». (Sontag 52) Dans la science-fiction comme dans la pornographie, on retrouverait un même rapport au temps et à l'espace, les mêmes « paysages anhistoriques » et « actions figées dans le temps»: « The ahistorical dreamlike landscape where action is situated, the peculiarly congealed time in which acts are performed - these occur almost as often in science fiction as they do in pornography ». (Sontag 46) Par ailleurs, le « Et si...? » scientifique et science-fictionnel (cf. Wright) n’est pas sans rappeler la froide « capacité démonstrative » que Deleuze attribue à Sade (cf. Deleuze 26).

Il s'est créé une sorte de sous-genre autour de la SF sadomasochiste, mais le phénomène demeure surtout cantonné au domaine anglosaxon, à un point tel que des auteures telles que la belge francophone Chelsea Shepard choisissent de faire carrière en anglais ${ }^{9}$. Cependant, ailleurs dans le corpus de science-fiction, des éléments SM apparaissent parfois sous forme de thèmes. La présence du sadomasochisme dans la science-fiction semble être à la fois un sous-genre, et un topos récurrent dans la science-fiction en général. Pour les besoins de cet article, j'ai choisi trois œuvres de science-fiction dépeignant des corps souffrant d'une manière qui fraie avec le sadomasochisme. Il ne s'agit pas de textes pornographiques, ni même de fictions érotiques. De plus, ces textes appartiennent au domaine de la science-fiction québécoise où le sous-genre de la SF SM est à peu près inexistant. Cependant, dans ces récits, les corps sont réifiés tout en étant mis à nus et utilisés, et la douleur est, d'une certaine façon, érotisée d'une manière qui s'apparente aux pratiques sadomasochistes ou SM (ou S/M ou S\&M) ou $\operatorname{BDSM}^{10}$, que Pat Califia décrit ainsi :

S\&M is scary. That's at least half its significance. We select the most frightening, disgusting, or unacceptable activities and transmute them into pleasure. We make use of all the forbidden symbols and all the disowned emotions. S\&M is a deliberate, premeditated erotic blasphemy. It is a form of sexual extremism and sexual dissent. (Califia 140)

Dans les trois récits que j'ai choisis pour cette étude, de manière différente mais convergente, les personnages sont soumis à un traitement qui à la fois génère un inconfort et une douleur, et éveille chez eux un plaisir ambigu. «La carte du tendre », une nouvelle d'Élisabeth Vonarburg, sans doute la plus connue des auteures québécoises de SF, décrit comment un personnage, appelé simplement le « Sujet » se fait littéralement sculpter sur scène par un autre personnage appelé l'« Opérateur ». Dans le roman Lame d’Esther Rochon, le personnage 
éponyme est envoyé aux enfers (au pluriel, sans la majuscule) après sa mort et se voit condamné à être exposé à la vue de tous dans un état progressif de dégradation. La mue de I'hermaphrodite, le premier roman de Karoline George, repose aussi sur le principe de I'humiliation par la voie d'une réification du corps : dans ce récit, Hermany, une créature hermaphrodite, accepte en guise de châtiment pour un crime qu'elle a commis, de livrer publiquement toute son histoire sur le cyber-réseau et d'être ainsi jugée sur la base des cotes d'écoute. Alors que chez Karoline Georges, le malaise est à dominante psychologique (hormis la nudité imposé à Hermany), les personnages de Vonarburg subissent essentiellement une douleur physique, et la situation imaginée par Esther Rochon est à peu près à mi-chemin. De plus, les trois histoires ont un autre trait commun : la souffrance y est en grande partie un état que les personnages ont choisi, consciemment ou inconsciemment, de manière relative ou absolue. Lame dans le roman de Rochon se voit imposer une damnation éternelle qui n'est que la suite de la façon dont elle vivait sa vie précédente (les enfers, chez l'auteure, ne sont rien d'autre qu'un univers parallèle au nôtre). L'hermaphrodite du roman de Georges a choisi ce châtiment public plutôt qu'une sentence plus traditionnelle. Et tant le Sujet que l'Opérateur dans la nouvelle de Vonarburg sont des performers, et le jeu sadomasochiste est l'art qu'ils pratiquent. Les trois textes ont au moins un autre élément en commun : ils présentent tous la souffrance de corps féminins (ou de corps qui s'identifient à un moment ou à un autre comme tels) dans le contexte d'une performance : une confession publique pour Hermany, un spectacle devant auditoire pour l'Opérateur et le Sujet, une damnation éternelle à la vue de tous pour Lame.

En matière d'interactions sadomasochistes, la question des rôles préétablis (homme/femme, sadique/masochiste) n'est pas anodine; elle nous arrive chargée de sens, tant du côté de la psychanalyse que des théories féministes. Ces récits que j'ai choisi d'étudier ici, en tant que systèmes énonciatifs fictifs, semblent cependant dépasser les limites des oppositions binaires supposant un masochisme féminin et un sadisme masculin. Observant comment et surtout à partir de quelle perspective ces scènes sadomasochistes se déploient, je vais essayer de montrer comment les thèmes et structures liés au BDSM travaillent ces textes à différents niveaux narratifs. Combinant entre autres l'analyse du discours narratif, les théories psychanalytiques du sadomasochisme, et I'étude de la performance BDSM, non seulement vais-je considérer ce que la science-fiction nous dit de la sexualité et du sadomasochisme, mais je tenterai de voir ce que le sadomasochisme, en tant que cadre d'analyse, peut nous apprendre à propos de l'écriture de science-fiction et, pourquoi pas, à propos de l'écriture en général.

\section{Une cartographie de la douleur}

Le roman de Karoline Georges s'amorce par la condamnation du personnage principal. Hermany est un être hermaphrodite. Sa narration est au féminin, car le personnage choisit une identité féminine après l'amputation de son pénis, de ses testicules, puis de sa vulve et de ses ovaires. Au début du récit, elle a blessé trente-trois 
personnes et presque tué le psychiatre qui l'avait aidée à canaliser ses pouvoirs paranormaux. On a alors donné le choix à Hermany : passer sa vie en prison ou se soumettre à une expérience judiciaire. Avant de se livrer à son bourreau, I'hermaphrodite demande si le châtiment impliquera de la torture et le juge répond à côté : « Nous ne sommes pas au Moyen Âge. »(Georges 12) S'il y a bien torture, psychologique surtout, on pourrait qualifier celle-ci de technologique: tandis que la condamnée raconte son histoire, son geôlier la tient informée des cotes d'écoute ou « captations » et la menace des pires conséquences si elle n'arrive pas à se rendre suffisamment intéressante. Non seulement doit-elle se mettre à nu, métaphoriquement et littéralement, mais elle doit garder la cote. Lorsque Hermany tente de se soustraire aux regards car, comme elle le dit, « [s]a mise à nu est d'une impeccable indécence » (Georges 77), on la prévient : «Règlement numéro 1C: tu dois exposer ta nudité en tout temps, sauf pendant les heures alouées au sommeil ». (Georges 77) Et si, parfois, elle essaie de s'évader dans l'ironie, on la prévient, en la traitant comme une simple machine: « Nous te suggérons d'éviter toute forme de cynisme. À moins qu'un programme masochiste ne dicte ton comportement ». (Georges 15) Tout est mis en œuvre pour plonger le personnage dans le malaise, depuis la nudité imposée, jusqu'au sommeil contrôlé, en passant par les menaces.

Le roman d'Esther Rochon s'amorce après la condamnation aux enfers du personnage éponyme et débute avec la description de la souffrance de Lame. Dans les enfers mous de Rochon, les damnés sont constamment en quête de satisfaction des sens, et en même temps dotés de corps impossibles à satisfaire. Lame est donc condamnée à subir les affres d'un appétit et d'une libido inextinguibles dans un corps destiné à enfler jusqu'à en perdre toute apparence humaine. Elle doit donc manger et se masturber sans arrêt. Les premières pages du livre décrivent sa douleur, son corps devenu étranger, son humiliation et sa dégradation.

Pourtant, ce monde-ci, horrible comme elle-même l'avait été en ville, atroce comme elle le méritait, lui convenait. Comme elle y souffrait! Comme tous y souffraient! Les corps y étaient malléables, devenant graduellement plus monstrueux, plus ignobles, impossibles à tuer, de plus en plus difficiles à mouvoir. On pourrait dire que c'était l'enfer. (Rochon 1)

Dans un tel état de déchéance, en tant que réceptionniste, nue, elle est exposée à la vue de tous, à titre d'exemple : « Les nouveaux arrivants étaient horrifiés en I'apercevant » (Rochon 4). Lame, cependant, à l'instar d'Hermany, est masochiste. «Elle aimait ses épines » (p. 7) lit-on à propos de sa vie sur Terre. Elle considère sa damnation de la même manière que le font ses tortionnaires : de façon froide et analytique, avec la même distance et la même fascination morbide. D'une certaine manière, elle s'est attiré ce châtiment qui n'est que le prolongement de sa vie antérieure :

Là-bas, elle avait préféré se faire mal. Se blesser le ventre, justement, ou le sexe. S'humilier en portant des vêtements trop serrés et en regardant ensuite les marques qu'ils laissaient. Il lui avait semblé mériter de souffrir, puisqu'elle était laide, indésirable, indigne de vivre. (Rochon 16) 
Au contraire des deux autres récits, dans la nouvelle de Vonarburg, la souffrance n'est pas une punition mais une vocation. Tant le Sujet que I'Opérateur sont des artistes et leur interaction sadomasochiste est une danse qu'ils réalisent au profit de leur public. L'Opérateur dévoile lentement le Sujet, retirant peu à peu des couches d'une créature qui prend d'abord la forme d'un cube noir, puis d'un cristal, puis qui apparaît finalement comme une jeune femme nue. Aussitôt que le Sujet prend une allure humaine, il commence à être question de douleur dans ce qui est appelé « le Grand Jeu »(p. 175). En effet, le processus ne s'arrête pas là : graduellement, la peau est détachée du corps du Sujet cependant que le même traitement est imposé désormais au corps de I'Opérateur. La description de la scène est explicite :

Avec un petit bruit de déchirement discret, les ongles sont arrachés de l'extrémité des phalangettes invisibles sous l'épaisseur de la chair. Une autre petite ventouse vient se coller à chacun des doigts comme une bouche, aspirant le sang qui sourd de la circonférence de l'ongle, puis cautérisant les vaisseaux sanguins après avoir injecté dans le même mouvement un analgésique local à effet retardé. Le hurlement du Sujet s'interrompt donc bientôt. (Vonarburg $\left.175^{11}\right)$

La scène est d'une extrême précision: combien d'incisions à effectuer, comment retirer la peau, comment s'y prendre avec chaque partie du corps - en particulier les parties sexuelles:

Il y a des zones particulièrement délicates, celles où la peau est plus fine (face interne du poignet, creux du bras, aisselles, aréoles... Ou, bien sûr, de l'autre côté, le creux poplité, l'aine et, lorsque le Sujet est un homme, le pénis, lorsqu'il est traité initialement comme un doigt: il faut remonter du gland à la racine en passant le surplomb du prépuce, et venir à bout de la mollesse du scrotum). (Vonarburg 177)

À mesure que la performance fictive, de même que le récit, progresse, il devient de plus en plus évident que tout dans ce spectacle sadomasochiste repose sur le contrôle, sur la capacité à supporter la douleur, sur l'aptitude à mener le projet à bien.

Peut-on assimiler ces trois échanges sadomasochistes à autant de scènes BDSM? Tout au moins les trois situations ont-elles une teneur sexuelle et évoquent-elles une certaine gratification érotique. Dans La mue de I'hermaphrodite, après un spectacle dramatique impliquant des morts et des corps mutilés - hologrammes ou sacrifices véritables? - le père adoptif d'Hermany - le psychiatre qu'elle tentera plus tard d'assassiner — lui demande : «As-tu ressenti cette fascination viscérale, ce mélange complexe de répugnance et de curiosité, cette empathie nappée de distanciation? » (Georges 90) Ce à quoi elle répond non dans le dialogue, mais dans le fil de sa narration: «Oui, j'avais éprouvé ce mélange complexe. Oui, c'était une expérimentation d'une puissance unique. Et c'était exactement ce qui me perturbait ». (Georges 90)

Dans Lame, le personnage principal est excité par sa propre humiliation et est gardé dans un état constant de désir sexuel. Sa satisfaction sexuelle est toujours différée car, en tant que réceptionniste des enfers, elle s'impose de préserver un peu de dignité; et puisqu'elle conserve son emploi plus longtemps que celles qui l'ont précédée (qui ont été plus vite précipitées dans la déchéance physique), on peut en déduire que le report de la jouissance érotique (et non l'abstinence) est en soi pour elle une certaine forme de gratification. Cela bien sûr nous 
ramène au jeu sadomasochiste où le contrôle du plaisir de l'autre est une notion récurrente, du moins si l'on en croit les guides des pratiques BDSM (voir Brame et alii 110-111), voire les fictions regroupées sous cette étiquette.

Dans « La carte du tendre », l'attention demeure concentrée à l'extérieur des deux personnages qui livrent leur prestation. Le lecteur n'a pas accès à leurs pensées, seulement à leurs actions. Le narrateur suggère pourtant que leur scène leur procure une certaine satisfaction sexuelle, ne serait-ce que celle liée à l'investissement libidinal des créateurs dans leur création : « sa façon de tourner autour de l'enveloppe du Sujet, puis de la sienne, indique assez clairement sa satisfaction devant l'issue de la confrontation: ils ont été, en quelque sorte, dignes l'un de l'autre ». (Vonarburg 179) La présence d'un spectateur et d'une spectatrice, et de leur interaction amoureuse, ajoute cependant une dimension érotique à la scène: unis dans un rapport pour le moins sensuel, ils s'embrassent et se caressent l'un l'autre tout en observant la scène: « Posée sur la table entre les deux verres à longue tige, la main de l'homme enveloppe la main de la femme; la tête de la femme est inclinée sur l'épaule de l'homme. Ils regardent tous les deux la scène circulaire, en contre-bas ». (Vonarburg 174) L'interaction entre I'Opérateur et le Sujet évoque un peu la notion du SSC -Safe, Sane, and Consensual (Sûr, sain, et consensue/) qui sert de credo à une part importante de la communauté BDSM (voir Miller 2002) : dans ce jeu qui a fait l'objet d'une négociation préalable, personne ne sera blessé de manière irréversible ni surtout au-delà de ce à quoi les parties ont consenti. La présence du couple de spectateurs prolonge ce principe d'une cruauté sans conséquence (les joueurs n'en garderont aucune trace) lorsque I'on comprend qu'ils ne sont autres que le Sujet et I'Opérateur assistant à leur propre performance présentée en reprise :

Des exclamations indiquent ici et là que leurs noms sont familiers à plusieurs spectateurs. Pendant un moment, dans certaines alcôves, les spéculations vont bon train sur ce qui a bien pu pousser le Gérant à présenter un spectacle vieux déjà, si on se rappelle bien, d'une dizaine d'années; les artistes sont depuis longtemps partis chacun de son côté vers d'autres destinées et d'autres formes d'art plus modernes. (Vonarburg 80)

\section{Une performance de la douleur}

Ces trois récits apparaissent comme des mises en scène du désir humain. Lynda Hart soutient, à propos de la performance sadomasochiste et du désir en général : « desire is always in some sense "theatrical" ». Un peu plus loin, elle ajoute : «[d]esire, like theatre, takes place in the fantasy one constructs with others ». (Hart 8-9) Le principe de la performance est également intéressant en ce qu'il insiste plus sur les moyens que sur la fin, contribuant ainsi à la dynamique de la représentation, et à la déconstruction d'un « sujet en procès » ${ }^{12}$.

L'enjeu ici n'est pas tant la pseudo-réalité de I'histoire, mais sa faculté de retenir l'attention du public. L'histoire d'Élisabeth Vonarburg, à l'instar de celle de Karoline Georges qui insiste sur les cotes d'écoute, inclut d'ailleurs des références à la nature divertissante du spectacle. Avant qu'il ne devienne évident qu'il s'agit ici d'une rediffusion holographique, le récit insiste sur le fait que la représentation de ce soir-là est sujette à d'infinies 
variations, notamment parce que les spectateurs jouent un rôle dans son déroulement. Ils applaudissent, poussent des exclamations, et peuvent formuler des commentaires auprès du gérant par la voie du réseau de communication :

Un spectateur plus perspicace que les autres se met à applaudir dans le cinquième gradin; d'autres comprennent aussi, quelques secondes plus tard, et bientôt le reste de l'assistance en fait autant - par effet d'entraînement ou soudaine illumination, impossible d'en décider. (Vonarburg 179)

Chacun à sa manière, ces trois récits comportent plus d'un niveau narratif. Dans le roman de Karoline Georges, comme on l'a vu, il s'agit carrément d'une mise en abyme: le récit des événements qui ont mené à la condamnation d'Hermany est entrecoupé par un dialogue, au temps présent, en caractères gras, entre la prisonnière et son tortionnaire cybernétique. Dans Lame, il y a aussi un certain élément de mise en abyme puisque, au milieu du récit à la troisième personne racontant l'histoire de Lame, on retrouve un second récit, remis à Lame sous la forme de feuillets, et décrivant, toujours à la troisième personne, l'arrivée d'un autre personnage aux enfers. Dans le cas de la nouvelle de Vonarburg, s'il n'y a pas à proprement parler d'enchâssement des récits, il y a tout de même une double focalisation : celle sur le spectacle et celle sur les spectateurs (en italiques).

Cette mise à distance des scènes souligne qu'il s'agit de construction, de scénarios, de mises en scène. Encore ici, cela nous ramène à la scène BDSM. La performance est en effet un élément important de l'échange de pouvoir sadomasochiste et, en tant que force structurante dans ce genre de récits, elle fait office de trope. Dans les trois textes vus ici, cela renforce l'idée de la fiction comme processus plutôt que comme objet fini. Les références au public contribuent également à inclure le lecteur et la lectrice dans la situation discursive. Dans le contexte de la représentation de l'identité sexuelle et de la sexualité, la mise en scène de la performance s'avère également fructeuse considérant que, selon Judith Butler, celle-ci, de manière générale, précède souvent la production du gender (Butler 1990 339) - ce qui n'est qu'une autre façon d'énoncer le « On ne naît pas femme, on le devient » beauvoirien. L'inclusion de la performance dans le tissu narratif lui-même apparaît comme une façon de souligner sa portée politique en fonction de la construction de nouvelles expressions liées au genre. En effet, il ne faut pas oublier que, dans les trois récits, il y a une transformation de l'enveloppe physique des personnages: dans la nouvelle de Vonarburg l'opérateur revêt littéralement la peau du sujet et vice versa; dans le roman de George, le personnage hermaphrodite perd ses attributs sexuels jusqu'à prendre une apparence asexuée - sans cesser d'adopter le pronom féminin — ; dans le roman de Rochon, Lame passe de son corps informe de damnée tenaillée par une faim de sexe et de nourriture à un corps de jeune femme désirée, et il en va de même, en sens inverse, pour Vaste qui l'a sauvée et qui se voit soudain condamné aux chauds enfers :

Ses yeux, ses oreilles, ses narines, tout était comme couvert d'un voile de peau cornée. Ses doigts et ses orteils étaient attachés entre eux, comme enrobés d'une couenne rousse. Il n'avait plus de cheveux. Son sexe était bien en vue, probablement pour lui procurer un maximum de souffrance. (Rochon 108) 
Or, dans la scène BDSM, la performance favorise l'exploration d'un éventail de rôles dans le contexte d'une rencontre sexuelle et de la constante re-création de l'interaction sexuelle. Et ces rôles, paradoxalement, permettent aux joueurs sadomasochistes de pénétrer plus intimement dans leur psyché et les aide à approfondir leur interaction avec l'autre. Lynda Hart se fonde sur l'expression anglaise « to have sex » et fait remarquer :

Sadomasochistic, or any kind of « perverse » sexuality is about doing. Whereas «straight » sex (whatever the preference of the people involved) is about having. Now, this is an obvious but, I think, crucial and fascinating difference. For, the notion of « having sex » signifies at once that « sex » is something one can own, and that it (sex) was there prior to the performance. The s/m sensualist, quite contrarily, in doing a scene makes sex in the performance. (Hart 1997 148)

\section{Une perversité protéiforme}

Puisque la performance BDSM permet de faire le sexe plutôt que de l'avoir, en va-t-il de même pour le genre? Lorsque ces auteures, et les instances narratives qu'elles construisent, représentent des corps féminins ou féminisés qui souffrent, perpétuent-elles l'image traditionnelle de la femme comme dominée voire martyre et donc les inégalités du système patriarcal, ou alors parviennent-elles à une appropriation de cette image par l'expression directe des fantasmes et peurs les plus extrêmes? Et si ces récits impliquent une interaction sadomasochiste, ou un échange à teneur BDSM, et si ces scènes fictives sont incluses dans le contexte d'une performance, où donc le sujet discursif (I'énonciateur principal) se situe-t-il?

Dans ces textes, les rôles apparemment binaires du sadomasochisme croisent la dichotomie masculin/féminin. Cela rejoint les origines mêmes des théories sur le sadisme et le masochisme. Sans passer en revue la théorie psychanalytique sur la question, disons de manière un peu schématique que, dans la tradition freudienne (et, dans une certaine mesure, deleuzienne), les femmes n'obtiennent que la portion congrue des fantasmes sadomasochistes. Freud considère en effet que seuls les hommes peuvent avoir accès à l'ensemble du spectre de ces fantasmes, qu'eux seuls en particulier font l'expérience de ce qu'il appelle le «masochisme féminin» puisque les femmes le vivent déjà... comme une réalité. Chez ces dernières, toujours selon Freud, le masochisme demeure donc moral, et non sexuel, puisqu'il n'est pas un état désirable mais bien réel. Comme le résume Lynda Hart, empruntant les concepts d'Austin, selon une telle logique, le masochisme féminin ne peut être que « constatatif » alors qu'il est pour les hommes un performatif (cf. Hart 1997 89).

D'une manière constatative plutôt que performative, force est de constater que certains aspects des trois récits étudiés ici appuient la dichotomie masochisme féminin/sadisme masculin. Dans « La carte du tendre », I'Opérateur est un homme et le Sujet se révèle comme étant une femme. La scène, pour une large part, met en opposition un personnage masculin actif sculptant un sujet féminin passif, ce qui semble renforcer les stéréotypes. Au niveau des deux observateurs principaux, on retrouve aussi plusieurs signes de soumission féminine à

I'homme : « [I]'homme lui prend le visage » (Vonarburg 73); « la main de I'homme enveloppe la main de la femme 
» (Vonarburg 73); «la tête de la femme est inclinée sur l'épaule de l'homme » (Vonarburg 173-174). De même, dans Lame, les enfers sont dirigés par un leader masculin, le roi Har, et Lame est rescapée des enfers mous par Vaste, un autre personnage masculin, puis par Rel, le prince héritier. Cela ne semble que reproduire une dynamique sexiste : même libérée, il semble d'abord qu'elle demeurera la propriété de son amant et sera soumise à sa cruauté tout comme, auparavant, elle appartenait aux enfers. Enfin, dans La mue de I'hermaphrodite, il est particulièrement éloquent que Hermany choisisse de se présenter comme un individu du sexe féminin ou, du moins, en usant du féminin grammatical. Aussi, le roman met peut-être en scène une créature qui possède les deux sexes, mais l'emploi des formes féminines conditionne la construction d'un personnage en grande partie féminin. On se retrouve donc avec un personnage (auto-)identifié comme féminin qui souffre aux mains d'un geôlier cybernétique désigné arbitrairement par des formes grammaticales masculines.

Cependant, chacun de ces récits comporte au moins un élément de cohabitation ou de renversement des genres sexuels, ce qui les empêche de sombrer tout à fait dans le binarisme identitaire. Lorsque Hermany, d'abord hermaphrodite puis créature asexuée (puisqu'on a dû l'amputer de ses organes sexuels) se désigne par le pronom féminin, cela nous rappelle que le genre est quelque chose de construit. Dans le roman de Rochon également on retrouve un personnage d'hermaphrodite, puisque le prince héritier est doté des deux sexes. De plus, on assiste à une inversion des rôles lorsque Lame a l'occasion de rendre la pareille à Vaste, et de le sauver des enfers où le roi I'a expédié. Chez Vonarburg, il est plutôt question de croisement puisque, vers la fin de la performance, I'Opérateur masculin enfile la peau du Sujet féminin et vice versa.

Considérant les liens que, dans son étude comparée de Sade et de Sacher-Masoch, Deleuze établit entre le sadisme, le masochisme, et la notion de Loi (appelée ailleurs par Lacan « Nom du père » - voir Chaumon 2004), on remarque dans ces textes des croisements fascinants. Un chiasme intéressant s'opère entre les deux premières histoires d'une part, et celle d'Élisabeth Vonarburg d'autre part. Le roman de Rochon est raconté à la troisième personne, mais I'histoire est focalisée sur Lame, la masochiste, la soumise ou bottom, pour ramener le vocabulaire BDSM dans la discussion. La mue de l'hermaphrodite, de son côté, est basé sur un dialogue entre un geôlier et sa prisonnière, mais la majeure partie du récit est consacrée au témoignage d'Hermany qui s'identifie comme être féminin et qui se décrit dans une position de soumission, de bottoming. Au contraire, dans la nouvelle de Vonarburg, alors que la description est aussi à la troisième personne, mais à focalisation externe, l'accent est mis sur les gestes de l'Opérateur (le dominant, le top) - durant une bonne partie de la performance, d'abord cube puis cristal, le Sujet n'est même pas posé comme un être vivant. Or Deleuze note que le masochiste insiste sur la « loi du contrat » (cf. Deleuze 80 ) et cultive le rite, tandis que le héros sadique « invente une nouvelle manière de remonter de la loi à un principe supérieur», à une «nature première destructrice de loi » (cf. Deleuze 79). On pourrait donc s'attendre à ce que les récits de Rochon et de Georges, insistant sur le point de vue du masochiste, soient plus axés sur le rite, sur la loi préétablie, et que, au contraire, la nouvelle de Vonarburg repose sur la loi 
fantasque du sadique. C'est pourtant le contraire qui se produit. Dans « La carte du tendre », la scène s'élabore dans les règles de l'art, selon un canevas bien précis (en anglais on dirait by the book), d'une façon qui l'associe plus aux postures masochistes. En revanche, La mue de I'hermaphrodite et Lame, qui semblent pourtant être focalisés sur des personnages masochistes, dépeignent une loi imprévisible, écrite mais dont le script peut être constamment modifié, du type de celles que Deleuze attribue au sadique. Ce chiasme suggère que les identités, bien que situées dans un contexte apparemment binaire, ne sont pas tout à fait comme on le croit, et pas tout à fait univoques.

Toutes ces ambivalences ou ambiguïtés contredisent, du moins en partie, l'idée d'une science-fiction (et peut-être d'une littérature pornographique, par la voie de la fiction sadomasochiste) qui ne serait que mécaniste. La scène BDSM est fondée sur des rôles prédéterminés certes, dans une structure soigneusement négociée, ce qui évoque l'idée de «paysages anhistoriques » et $d^{\prime}$ « actions figées dans le temps », tels que les décrits Susan Sontag comme relevant à la fois de la science-fiction et de la pornographie. Cependant, il s'agit d'un jeu auquel les participants choisissent d'adhérer de leur plein gré et d'une performance dans laquelle le processus est au moins aussi important que le résultat, et où le principe du choix (celui d'adhérer ou non au jeu) est central. Ces fictions, par toutes sortes de moyens narratifs (enchâssement des récits, focalisation variable), linguistiques (jeu sur le genre grammatical), voire actantiels (échange de rôles), par un jeu, justement, sur ce qui pourrait a priori sembler mécanique, bouleversant les rôles et les idées préconçues. Par ailleurs, le corps, dans ces fictions, est peut-être traité comme une machine et réduit à ses réponses les plus automatiques (comme beaucoup de corps sciencefictifs), mais l'interaction sadomasochiste, ici, se présente aussi comme un moyen de subvertir les réactions les plus naturelles, de court-circuiter les réactions de défense et de transformer la douleur en un plaisir ambigu - un plaisir esthétique tout au moins.

À cela, il semble pertinent d'ajouter qu'en se promenant ainsi à la frontière entre le sadisme et le masochisme, entre la domination et la soumission, entre topping et bottoming, ce genre de fiction semble aussi se situer entre l'exhibitionnisme et le voyeurisme, leurs auteures insistant sur des fantasmes qui ne sont peut-être pas les leurs, mais auxquels elles prêtent néanmoins leur plume et les lecteurs et lectrices s'en délectant et s'y identifiant, bien installés dans leur fauteuil de lecture préféré. Ainsi, dans un article sur le roman Misery, Douglas Keesey s'interroge à la fois sur la position du personnage principal Paul Sheldon, écrivain fictif de I'histoire et sur celle de l'auteur Stephen King. Il part du fait que le personnage d'écrivain, dans ce roman, s'identifie à la fois à son propre personnage de Misery, heroïne gothique, et à Annie, sa lectrice passionnée et sadique, et il note une ambivalence tant par rapport au genre sexuel que par rapport à la position de sadique ou de masochiste : « Considering the association of "weeping" with feminization in the novel, is there a certain amount of gender 
ambiguity at the end as well as ambivalence as to whether Paul is a triumphant sadist (a writer of fiction that can scare us) or still a suffering masochist? » (Keesey 66) Parce que ces récits sont construits autour de fantasmes, ils peuvent aussi être perçus comme un moyen de rejouer ceux-ci, un peu comme dans le processus psychanalytique, et donc comme un exutoire. Robert McClure Smith explique d'ailleurs combien les « scénarios fantasmatiques » peuvent être libérateurs lorsqu'ils se font à partir de conventions et d'archétypes :

Fantasy scenarios are specifically constructed in order to permit multiple and shifting identifications. [...] In short, re-presenting female masochism potentially disturbs the gendered polarities of dominance and submission on a number of different levels of textual representation. (McClure Smith 1-2)

Dans les trois récits que j'ai étudiés ici, le principe d'une performance et d'une mise en abyme multiplie en quelque sorte les possibilités d'identification en insistant sur la fiction comme création et en reproduisant la présence de l'auteure à la fois comme créatrice et spectatrice de sa propre scène ${ }^{13}$.

Le cadre d'analyse que j'ai élaboré pour cette recherche montre que les auteures de science-fiction - en fait, les auteurs en général - tout comme l'enfant freudien, sont d'authentiques pervers polymorphes. Lorsqu'elles adoptent la perspective du bottom, ou lorsqu'elles fondent leurs rapports sur la loi du contrat masochiste, ces auteures montrent combien, en position d'écriture, elles peuvent être sadiques avec leurs personnages - comme l'enfant dans ses jeux. Les auteures donnent accès à leur lecteur ou lectrice à une imagerie qui est peut-être ou non la leur, tout comme les auteurs de romans noirs n'ont pas tous d'obscurs fantasmes assassins; comme le dit Beauvoir à propos de Sade : «Ce n'est pas par le meurtre que s'accomplit l'érotisme de Sade : c'est par la littérature. » (p. 45) Ces auteurs, cependant, doivent assumer ces images en tant qu'écrivains - et tant mieux si, aujourd'hui, c'est rarement au péril de leur liberté. D'une certaine manière, en écrivant, elles se mettent et peutêtre nous placent dans la même position de vulnérabilité, dans le même type de performance qu'elles imposent à leurs personnages lorsqu'elles les jettent dans le ring BDSM.

http://www.brocku.ca/cfra/voixplurielles05-02/index.html 


\section{Références}

Allison, Dorothy. Peau. Traduit de I'anglais par Nicolas Milton. Paris: Balland, 1999.

Austin, John Langshaw. How to Do Things with Words. Cambridge: Harvard University Press, 1962.

Beauvoir, Simone de. « Faut-il brûler Sade? » Paris : Gallimard, 1955.

Bérard, Sylvie. «BDSMSF(QF): Sadomasochistic Readings of Québécois Women's Science Fiction ». Queer Universes: Sexualities in Science Fiction. W.G Pearson, V. Hollinger et J. Gordon (dir. publ.). Liverpool: Liverpool University Press, 2005. 180-198.

Bozzetto, Roger. «Esther Rochon: L'Emergence d'une écrivaine de SF au Québec dans les années 80 ». Novelli, Novella (Ed.). Au cœur de l'avenir. Littérature d'anticipation dans les textes et à l'écran. Actes du Séminaire international de L'Aquila (29-30 septembre 2000). [s.I.]: Angelus Novus Edizioni, 2002.

Brame, Gloria G., William D. Brame and Jon Jacobs. Different Loving. The World of Sexual Dominance \& Submission. New York: Villard Books, 1995.

Braudy Leo. « Fanny Hill and Materialism ». Eighteen-Century Studies, 4.1 (1970). 21-40.

Butler, Judith. «Performative Acts and Gender Constitution: An Essay in Phenomenology and Feminist Theory ». Sue-Ellen Case (Ed.). Performing Feminisms. Baltimore: The Johns Hopkins University Press, 1990. 27072.

Butler, Octavia E. « Bloodchild». Pamela Sargent. Women of Wonder: The Contemporary Years: Science Fiction by Women from the 1970s to the 1990s. New York: A Harvest Original. 1995 [1984], 123-140.

Cadigan, Pat. Synners. New York: Bantam, 1991.

Califia, Pat.« A secret side of lesbian sexuality ». S\&M. Sudies in Dominance and Submission. Thomas S. Weinberg (dir. publ.). New York: Prometheus Books, 1995. 139-149.

Chancer, Lynn S. Sadomasochism in Everyday Life: The Dynamics of Power and Powerlessness. New Brunswick: Rutgers University Press, 1992.

Delany, Samuel. "Aye, And Gomorrha" and Other Stories. New York: Vintage Books, 2003.

Deleuze, Gilles. Présentation de Sacher-Masoch. Paris: Minuit, 1967.

Freud, Sigmund Freud. «Sadism and Masochism ». Weinberg, Thomas S. (dir. publ.). S\&M. Studies in Dominance \& Submission. Amherst, (NY): Prometheus Books, 1995. 33-35.

Frontain, K.M. Bound in Stone. [S.I.]: Thinkharder Press, 1985.

Georges, Karoline. La mue de I'hermaphrodite. Montréal: Leméac, 2001.

Hart, Lynda. «That Was Then: This Is Now: Ex-Changing the Phallus ». Postmodern Culture 4.1 (1993).

Hart, Lynda. Between the Body and the Flesh. Performing Sadomasochism. New York: Columbia University Press, 1997.

Keesey, Douglas. « Your Legs Must Be Singing Grand Opera. Masculinity, Masochism, and Stephen King's Misery ». American Imago, 59.1 (2002). 53-71.

Kerbrat-Orecchioni, Catherine. L'énonciation: De la subjectivité dans le langage. Paris: Armand-Colin, 1980.

Lacan, Jacques. Écrits. Paris: Seuil, 1966.

Latham, Rob. «Sextrapolation in New Wave Science Fiction. Science Fiction Studies 33.2 (juillet 2006). $251-274$.

Le Guin, Ursula K. The Language of the Night: Essays on Fantasy and Science Fiction. New York: Harper Collins Publishers, 1989.

Macintyre, Ben. « Of human bondage: books can lead to a novel sort of enslavement. » The Times (United Kingdom), May 27, 2006. 20.

Massé, Michelle A. In the Name of Love: Women, Masochism, and the Gothic. Ithaca: Cornell University Press, 1992.

McCaffrey, Anne. The Ship Who Sang. New York: Ballantine, 1970.

Melzer, Patricia. Queer Universes: Sexualities in Science Fiction. W.G Pearson, V. Hollinger et J. Gordon (dir. publ.). Liverpool: Liverpool University Press, 2008. 160-179.

Midori. Wild Side Sex. The Book of Kink. Los Angeles: Daedalus Publishing, 2005. 
Miller, Elaine and Gary Switch. «Safe, Sane, and Consensual -- SSC -- Risk-Aware Consensual Kink -- RACK VancouverLeather.Com » Vancouver Leather. <http://vancouverleather.com/bdsm/ssc_rack.html>. 2002 Page consultée le 16 juillet 2008.

Reinelt, Janelle G. «The Politics of Discourse: Performativity meets Theatricality'. SubStance, $31.2 \& 3$ (2002). 201215.

Rochon, Esther. «Présentation subjective des Chroniques infernales. « Novelli, Novella (Ed.). Au cœur de l'avenir. Littérature d'anticipation dans les textes et à l'écran. Actes du Séminaire international de L'Aquila (29-30 septembre 2000). [n.p.]: Angelus Novus Edizioni, 2002.

Rochon, Esther. Lame. Montréal: Québec-Amérique, 1995.

Schaeffer, Jacqueline. « Une perversion au féminin? À la folie! » Filigrane, 12.2 (automne 2003). 66-79.

Shepard, Chelsea. Two Moons. Book One. Worthy of a Master. Lincoln: iUniverse, 2003.

Shepard, Chelsea. Two Moons. Book Two. The Freeman's Captive. Lincoln: iUniverse, 2004.

Sontag, Susan. «The Pornographic Imagination. «Styles of Radical Will. New York: Anchor Books, 1991 [1969].

Tan, Cecilia, «Sadomasochism and Science Fiction: Connections and Parallels» <http://www.ceciliatan.com/smsf.html>. 10 octobre 1995. Page consultée le 16 juillet 2008.

Tan, Cecilia, The Velderet. [S.I.]: Lulu Press, 2001.

Turner, Alice K. The Playboy Book of Science Fiction. New York: Harper Collins, 1998.

Vonarburg, Élisabeth. «La carte du tendre ». André Carpentier (Ed.). Aimer. Montréal: Quinze, $1986.163-181$. Ailleurs et au Japon. Montréal: Québec/Amérique, 1991.

Wright, Sean. New Wave of Speculative Fiction: The What If Factor. London; Crowswing Books, 2005. 
${ }^{1}$ La recherche dont fait état le présent article a d'abord fait l'objet d'une publication en anglais (voir Bérard 2008)

${ }^{2}$ Cf. Octavia Butler, « Bloodchild», 1984.

${ }^{3}$ Cf. Anne McCaffrey, The Ship Who Sang, 1961.

${ }^{4}$ Cf. Samuel Delany, « Aye, And Gomorrah », 1967.

${ }^{5}$ Cf. Pat Cadigan, Synners, 1991.

${ }^{6}$ Parfois, comme dans les exemples susmentionnés, l'exploration des limites du corps s'accompagne d'une réflexion sur l'identité, et en particulier sur l'identité sexuelle (gender), comme en témoignent, du côté anglosaxon, les nombreuses entrées de dictionnaire et d'encyclopédies consacrés au genre, de même que plusieurs anthologies thématiques. Par exemple, voir l'anthologie des textes primes dans le cadre des Tiptree Awards décernés chaque année à des œuvres explorant les questions d'identités sexuelles ou permettant de mieux comprendre celles-ci: Flying Cups and Saucers: Gender Explorations in Science Fiction and Fantasy (Cambridge: Edgewood, 1999). Voir aussi les anthologies suivantes: Joseph Elder (dir. publ.), Eros in Orbit. A Collection of all new science fiction stories about sex (New York: Pocket Books, 1974); Pamela Sargent (dir. publ.), Women of Wonder, the Classic Years: Science Fiction by Women from the 1940s to the 1970s (New York: Harvest, 1995); Pamela Sargent (dir. publ.), Women of Wonder, the Contemporary Years: Science Fiction by Women from the 1970s to the 1990s (New York: Harvest, 1995); Ellen Datlow (dir. publ.), Off Limits: Tales of Alien Sex (New York: Ace, 1997).

7 Dans un article portant sur la sexualité queer dans la science-fiction, Patricia Melzer décrit ainsi les livres de Cecila Tan: «Tan's books address various intersecting reading communities; they often include queer and/or transgender characters, all are hardcore erotica, most include S/M practices, and many are written within a science fiction setting. »] (Melzer 162)

8 "I am no longer surprised when I go to a play party at someone's house and find their book collection looks very similar to mine. A small shelf of Pat Califia, Anne Rice, The Story of $O$, of course, but also shelves and shelves of science fiction and fantasy. I am no longer surprised when I meet people at Renaissance fairs or science fiction conventions, and discover I already know them from the S/M community. Or vice versa. "There's so much crossover", people say. But I think there is no "crossover". We have always all been one. » (Tan)

9 Books such as Cecilia Tan's The Velderet (2001), Chelsea Shepard's Two Moons series (2003-2004), and K.M. Frontain's Bound in Stone series (2005) fall in that category.

10 «BDSM » est un acronyme polysémique en langue anglaise et dont les lettres signifient à la fois « bondage » (ou ligotage) et « discipline», « domination » et « soumission » et sadomasochisme. Il est apparu au début des années quatre-vingt-dix pour remplacer « $\mathrm{S} / \mathrm{M} \gg$ (ou simplement « SM »), jugé trop réducteur.

${ }^{11}$ La nouvelle a connu une réédition en 1991. Cependant, ce passage, comme tous les autres qui suivront, sont tirés de l'édition de 1986.

12 « "Performance" has been used to differentiate certain processes of performing from the products of theatrical performance, and in its most narrow usage, to identify performance art as that which, unlike "regular" theatrical performances, stages the subject in process, the making and fashioning of certain materials, especially the body, and the exploration of the limits of representation-ability ». (Reinelt 200)

${ }^{13}$ Lynda Hart fait remarquer que le fait que la performance soit fondée sur les vraies choses est essentiel dans une scène BDSM. Le public veut savoir que le jeu n'est pas truqué, qu'il implique de la vraie douleur et du vrai sang. Elle a étudié le travail de plusieurs performers et noté que la première question que posent les spectateurs est: « Est-ce que vous le faites pour vrai? » Le référent est, d'une certaine manière, nécessaire (voir Hart 1997241 ). Parce qu'ils mettent en scène leur propre corps, leur propre moi, les performers fictifs de ces trois récits, tout comme les performeurs SM que décrit Hart, vont au-delà de l'illusion de la référentialité. 